\title{
Anomalous transport and holographic momentum relaxation
}

\author{
Christian Copetti, ${ }^{a}$ Jorge Fernández-Pendás, ${ }^{a}$ Karl Landsteiner ${ }^{a}$ and Eugenio Megías ${ }^{b}$ \\ anstituto de Física Teórica UAM/CSIC, \\ c/ Nicolás Cabrera 13-15, Cantoblanco, Madrid, 28049 Spain \\ ${ }^{b}$ Departamento de Física Teórica, Universidad del País Vasco UPV/EHU, \\ Apartado 644, Bilbao, 48080 Spain \\ E-mail: christian.copetti@uam.es, j.fernandez.pendas@csic.es, \\ karl.landsteiner@uam.es, eugenio.megias@ehu.eus
}

ABSTRACT: The chiral magnetic and vortical effects denote the generation of dissipationless currents due to magnetic fields or rotation. They can be studied in holographic models with Chern-Simons couplings dual to anomalies in field theory. We study a holographic model with translation symmetry breaking based on linear massless scalar field backgrounds. We compute the electric DC conductivity and find that it can vanish for certain values of the translation symmetry breaking couplings. Then we compute the chiral magnetic and chiral vortical conductivities. They are completely independent of the holographic disorder couplings and take the usual values in terms of chemical potential and temperature. To arrive at this result we suggest a new definition of energy-momentum tensor in presence of the gravitational Chern-Simons coupling.

Keywords: AdS-CFT Correspondence, Anomalies in Field and String Theories, Holography and condensed matter physics (AdS/CMT)

ARXIV EPRINT: 1706.05294 


\section{Contents}

1 Introduction 1

2 Holographic momentum relaxation $\quad 2$

2.1 Holographic energy-momentum tensor 4

3 Electric and chiral magnetic conductivities $\quad 6$

$\begin{array}{ll}3.1 \text { DC conductivity } & 6\end{array}$

$\begin{array}{lll}3.2 & \text { Chiral magnetic conductivity } & 7\end{array}$

$\begin{array}{lll}3.3 & \text { Chiral vortical conductivity } & 8\end{array}$

4 Conclusion and discussion $\quad 9$

\section{Introduction}

At finite temperature and chemical potential anomalies give rise to new dissipationless transport phenomena, the chiral magnetic (CME) and chiral vortical effects (CVE) (see [1, 2] for recent reviews). Holography has played a major role to arrive at the modern understanding of these effects [3-6].

In holography the dynamics of strongly coupled quantum systems is mapped onto the gravitational dynamics of higher dimensional black holes $[7,8]$. In its most common form the dynamics of the black hole horizon describes a fluid and this horizon fluid can be mapped to the hydrodynamics of the dual strongly coupled quantum system. In the hydrodynamic setup momentum is an exactly conserved quantity. Momentum conservation gives rise to convective transport of charge and leads to infinite DC conductivities. Having applications of holography to strongly coupled condensed matter systems in mind, one wants however a setup in which momentum is not conserved resulting in finite DC conductivities. This means that translation symmetry must be broken. A way of doing this in holography is by giving the graviton a mass [9-13]. A particular simple way of introducing such a mass is based on massless scalar fields in AdS space with spatially linear profiles [14].

Such models have been analyzed in [15] for theories dual to $(2+1)$ dimensional strongly coupled field theories. There it was shown that the holographic DC conductivity obeys a lower bound of the form $e^{2} \sigma \geq 1$ where $e^{2}$ is the bulk Maxwell coupling. Therefore holographic disorder driven metal insulator transitions are absent. A different picture arises if additional couplings are included that lead to effective renormalization of the bulk gauge coupling constant. In $[16,17]$ it was found that couplings between the massless scalar fields and the Maxwell field lead to conductivities that are unbounded from below, even becoming negative for certain ranges of the parameters and thus indicating some instability of the system.

Here we will be interested to generalize the setup of $[16,17]$ to strongly coupled theories in $(3+1)$ dimensions described by holography. There are a couple of differences to the $(2+1)$ 
dimensional setup. First of all the scaling dimension of the conductivity changes. More importantly $(3+1)$ dimensional field theories with chiral fermions have chiral anomalies which in holography are represented by five dimensional Chern-Simons terms. Two such terms are relevant: a pure gauge Chern-Simons term for the usual chiral anomaly and a mixed gauge gravitational Chern-Simons term for the gravitational contribution to the anomaly. Inclusion of these terms is known to give rise to the chiral magnetic and chiral vortical effects (CME and CVE). These currents are a direct consequence of the anomalies and thus are protected from quantum corrections and give rise to dissipationless transport. One would therefore guess that their values have to be independent of the graviton mass and even the charged disorder parameter introduced in [16, 17]. The purpose of this work is to check this in a simple explicit example, and indeed we find that the CME and CVE are completely insensitive to the holographic disorder parameters. In order to arrive at this result we need however to re-analyze the form of the holographic energy-momentum tensor. For the particular case of the massless scalar field background under consideration the usual expression of the holographic energy-momentum tensor receives a correction due to the presence of the mixed gauge gravitational Chern-Simons term. Only by using this corrected form of the energy-momentum tensor we find the expected result that the anomalous transport is insensitive to the holographic disorder. This solves the puzzle found in [18] based on the uncorrected form of the energy-momentum tensor.

This work is organized as follows: we introduce the model and fix conventions in section 2. In section 3 we calculate the electric DC and the chiral magnetic and chiral vortical conductivities. We conclude and discuss our results in section 4.

\section{Holographic momentum relaxation}

We will base our considerations on the Stückelberg mechanism using massless scalar fields (Goldstone modes of translation symmetry breaking). For each spatial dimension we introduce a scalar field $X^{I}, I=1, \ldots, 3$. A spatially linear profile of these scalars provides the mass for the gravitons. The action of our model is

$$
S=\int d^{5} x \sqrt{-g}\left[R+12-\frac{1}{2} \partial^{\mu} X^{I} \partial_{\mu} X^{I}-\frac{1}{4} F^{2}-\frac{J}{4} \partial_{\mu} X^{I} \partial_{\nu} X^{I} F_{\lambda}^{\mu} F^{\lambda \nu}\right]+S_{\mathrm{GH}}+S_{\mathrm{CS}}+S_{\mathrm{CSK}} .
$$

For simplicity we have set the scale of the AdS radius, the gravitational coupling $\frac{1}{2 \kappa^{2}}$ and the bulk gauge coupling $e$ to one. The latter can easily be re-introduced by scaling the gauge field accordingly. The coupling $J$ represents the effects of disorder on the charged sector of the theory $[16,17]$. Our model is a particular simple case of the class of models studied there. $S_{\mathrm{GH}}$ is the usual Gibbons-Hawking counterterm. In addition we introduce a Chern-Simons action and counterterm

$$
\begin{aligned}
S_{\mathrm{GH}} & =2 \int_{\partial} d^{4} x \sqrt{\gamma} K, \\
S_{\mathrm{CS}} & =\int d^{5} x \sqrt{-g} \epsilon^{\mu \nu \rho \lambda \sigma} A_{\mu}\left(\frac{\alpha}{3} F_{\nu \rho} F_{\lambda \sigma}+\lambda R_{\beta \mu \nu}^{\alpha} R_{\alpha \lambda \sigma}^{\beta}\right), \\
S_{\mathrm{CSK}} & =-8 \lambda \int_{\partial} d^{4} x \sqrt{\gamma} n_{\mu} \epsilon^{\mu \nu \rho \lambda \sigma} A_{\nu} K_{\rho \tau} D_{\lambda} K_{\sigma}^{\tau},
\end{aligned}
$$


where $\gamma_{\mu \nu}$ is the induced metric and $K_{\mu \nu}$ is the extrinsic curvature on the boundary defined by an outward pointing unit normal vector. The gauge variation of the Chern-Simons action is a total derivative and leads to the anomaly

$$
\delta S=\int_{\partial} d^{4} x \sqrt{-\gamma} \epsilon^{i j k l}\left(\frac{\alpha}{3} F_{i j} F_{k l}+\lambda R_{b i j}^{a} R_{a k l}^{b}\right) .
$$

Here $R^{a}{ }_{b i j}$ is the intrinsic curvature on the boundary. This form holds for finite holographic cutoff only if the counterterm $S_{\mathrm{CSK}}$ is added to the action [6].

The equations of motion are

$$
\begin{aligned}
0= & G_{\mu \nu}-6 g_{\mu \nu}+\frac{1}{2} F_{\mu}{ }^{\lambda} F_{\lambda \nu}-\frac{1}{8} g_{\mu \nu} F^{2}-\frac{1}{2} \partial_{\mu} X^{I} \partial_{\nu} X^{I}+\frac{1}{4} g_{\mu \nu}\left(\partial_{\rho} X^{I}\right)\left(\partial^{\rho} X^{I}\right) \\
& -\frac{J}{4}(\tilde{X} . F . F+F . \tilde{X} \cdot F+F . F \cdot \tilde{X})_{\mu \nu}+\frac{J}{8} g_{\mu \nu} \operatorname{tr}(\tilde{X} . F . F)-2 \lambda \epsilon_{\alpha \beta \gamma \delta(\mu} \nabla_{\rho}\left(F^{\beta \alpha} R_{\nu)}^{\rho}{ }^{\gamma \delta}\right), \\
0= & \nabla_{\mu} F^{\mu \nu}+\frac{J}{2} \nabla_{\lambda}(\tilde{X} . F)^{\nu \lambda}-\frac{J}{2} \nabla_{\lambda}(\tilde{X} . F)^{\lambda \nu}+\epsilon^{\nu \alpha \beta \gamma \delta}\left(\alpha F_{\alpha \beta} F_{\gamma \delta}+\lambda R_{\sigma \alpha \beta}^{\rho} R_{\rho \gamma \delta}^{\sigma}\right), \\
0= & \square X^{I}+\frac{J}{2} \nabla_{\mu}\left(\partial_{\nu} X^{I} F_{\lambda}^{\nu} F^{\lambda \mu}\right) .
\end{aligned}
$$

Following [17] we have defined $\tilde{X}_{\mu \nu}=\partial_{\mu} X^{I} \partial_{\nu} X^{I}$ such that we can multiply $\tilde{X} . F . F=$ $\partial_{\mu} X^{I} \partial_{\nu} X^{I} F^{\nu}{ }_{\lambda} F^{\lambda \mu}$, etc. Momentum dissipation is implemented by giving the scalar fields a linear profile (note that there are only derivative couplings of $X^{I}$ ). We use three scalars for the three spatial dimensions and choose

$$
X^{1}=k x, \quad X^{2}=k y, \quad X^{3}=k z .
$$

Because the scalars couple only through derivatives, the field equations and solutions will still be formally translational invariant. The parameter $k$ gives the graviton however a mass and this suffices to make the DC conductivity of a charged black hole solution finite. As we will see, it also fixes a preferred frame (the rest frame of the impurity density) and no frame ambiguity in the definition of the anomalous transport coefficients appears [19, 20].

In order to define the background we look for charged black hole solutions with AdS asymptotics of the form

$$
\begin{aligned}
d s^{2} & =\frac{1}{u}\left(-f(u) d t^{2}+d x^{2}+d y^{2}+d z^{2}\right)+\frac{d u^{2}}{4 u^{2} f(u)}, \\
A_{t} & =\phi(u) .
\end{aligned}
$$

The equations of motion for the blackening factor and the time component of the gauge field are

$$
\begin{aligned}
& 0=f^{\prime}(u)+\frac{2}{u}(1-f(u))-\frac{u^{2}}{3}\left(\phi^{\prime}(u)\right)^{2}-\frac{k^{2}}{4}, \\
& 0=\phi^{\prime \prime}(u) .
\end{aligned}
$$


We fix two integration constants by demanding that $\phi$ vanishes at the horizon and that the horizon sits at $u=1$. The solutions are then

$$
\begin{aligned}
& f(u)=(1-u)\left(1+u-\frac{k^{2}}{4} u-\frac{\mu^{2}}{3} u^{2}\right), \\
& \Phi(u)=\mu(1-u) .
\end{aligned}
$$

We identify $\mu$ with the chemical potential. The temperature is

$$
T=\frac{1}{\pi}\left(1-\frac{k^{2}}{8}-\frac{\mu^{2}}{6}\right) .
$$

In this simple theory the background does not depend on the charge disorder coupling $J$. It therefore reduces to the solution found in [14].

\subsection{Holographic energy-momentum tensor}

The mixed gravitational Chern-Simons term makes the analysis of the holographic dictionary much more complicated than in the standard case. This is due in part to the fact that it is a higher derivative term. We want to treat it in an effective field theory spirit, i.e. in a perturbative way and compute the leading effects in the gravitational anomaly coupling $\lambda$. Even to this order we need to take into account that the standard definitions of the holographic operators might change. In particular the standard Brown-York stress tensor has to be supplemented with extra terms coming from the gravitational Chern-Simons action. Furthermore, the higher derivative nature of the theory entails that two independent modes emerge in the asymptotic expansion of the metric near the boundary. Following the standard dictionary these can be understood as sources for two different operators. However, it is in principle possible to impose asymptotic boundary conditions in which one of these modes is absent and the extrinsic curvature is a functional of the near-boundary metric. The variation of the on-shell action with respect to the boundary metric gives then the stress tensor of the dual theory.

Let us outline the main steps in the construction of the stress tensor in our case. For convenience we switch in this section to the standard Fefferman-Graham coordinates

$$
d s^{2}=d r^{2}+\gamma_{i j} d x^{i} d x^{j}
$$

where the asymptotic AdS expansion reads

$$
\gamma_{i j}=e^{2 r} \gamma_{i j}^{(0)}+\gamma_{i j}^{(-2)}+e^{-2 r} \gamma_{i j}^{(-4)}+\ldots
$$

Choosing a timelike hypersurface at a fixed $r$, we vary the on-shell action:

$$
\delta S=\frac{1}{2} \int_{\partial} \sqrt{-\gamma}\left(t^{i j} \delta \gamma_{i j}+u^{i j} \delta K_{i j}\right)+\delta S_{\text {matter }} .
$$

Notice that for now we keep $\gamma_{i j}$ and $K_{i j}$ as independent variable. Only at the end of the construction we will restrict ourselves to the asymptotic expansion (2.18). We will rephrase the bulk constraint equations as the Ward identities of the dual theory. This allows us to 
find a bulk expression for the stress tensor by comparison with the QFT expression. As we expect the theory to contain a further operator on general solutions, we will allow the extrinsic curvature to only enter, outside of the stress tensor, as a source term. Consistency however demands such contribution to vanish asymptotically once (2.18) is considered.

Using thus the standard forms of the variations $\delta \gamma_{i j}$ and $\delta K_{i j}$ under diffeomorphisms one finds the Ward identity:

$$
D_{i}\left(t^{i}{ }_{j}+u^{i l} K_{l j}\right)=u^{i l} D_{j} K_{i l}+F^{i j} J_{i}-Y^{I} \partial_{j} X_{I}-8 \lambda D_{k}\left(\epsilon^{l m n p} F_{l m} R^{k}{ }_{j n p}\right),
$$

where

$$
Y^{I}=\sqrt{-\gamma}\left[\dot{X}^{I}+\frac{J}{2}\left(F^{r i} \dot{X}^{I}+F^{j i} \partial_{j} X^{I}\right) F_{i r}\right]
$$

is the momentum conjugate to $X^{I}$ and

$$
J^{i}=\sqrt{-\gamma}\left[F^{i r}-8 \lambda \epsilon^{i j k l} K_{j}^{m} D_{k} K_{l m}+\frac{J}{2}\left(F^{i r} \partial_{i} X^{I} \partial^{j} X_{I}-\dot{X}_{I} \dot{X}^{I} F^{r j}-\dot{X}_{I} \partial_{i} X^{I} F^{i j}\right)\right]
$$

is the current. The latter fulfills the Ward identity

$$
D_{i} J^{i}=-\epsilon^{i j k l}\left(\alpha \sqrt{-\gamma} F_{i j} F_{k l}+\lambda R_{b i j}^{a} R_{a k l}^{b}\right) .
$$

To compare to a weakly coupled theory with $N_{\chi}$ chiral fermions we can identify

$$
\alpha=\frac{N_{\chi}}{32 \pi^{2}}, \quad \lambda=\frac{N_{\chi}}{768 \pi^{2}} .
$$

Notice that this definitions contain terms proportional to the couplings $J$ and $\lambda$ which vanish at the conformal boundary. Due to the fact that it obeys the correct form of Ward identity we define the holographic energy-momentum tensor as

$$
\Theta^{i}{ }_{j}=t^{i}{ }_{j}+u^{i l} K_{l j} .
$$

We also note that $\Theta^{i j}$ and $J^{i}$ are the covariant currents in the sense of [21] and (2.20), (2.23) are the covariant anomalies.

The tensor $t^{i j}$ can furthermore be divided into the standard Brown-York contribution $t_{0}^{i j}$ and a part that stems from the mixed gauge gravitational Chern-Simons term $t_{\lambda}^{i j}$. A lengthy but straightforward calculation gives

$$
\begin{aligned}
t_{0}^{i j} & =-2 \sqrt{-\gamma}\left(K^{i j}-K \gamma^{i j}\right), \\
t_{\lambda}^{i j} & =-8 \lambda \sqrt{-\gamma} \epsilon^{m n p(i}\left(2 D_{n} K_{p}^{j)} F_{r m}+\gamma^{j) l} \dot{K}_{l n} F_{p m}-F_{p m} K_{l}^{j)} K_{n}^{l}\right), \\
u^{i j} & =8 \lambda \sqrt{-\gamma} \epsilon^{m n p(i} F_{m n} K_{p}^{j)},
\end{aligned}
$$

where round brackets indicate symmetrization $A_{(i j)}=\frac{1}{2}\left(A_{i j}+A_{j i}\right)$.

The additional contributions will be essential to get the physically correct results for the anomalous transport coefficients. This generalizes the expression found in [22] to cases in which $\gamma^{(-2)}$ does not vanish. We also note that $\left(t_{0}\right)_{j}^{i}$ is divergent and needs to be 
regularized by the standard counterterms [23]. In contrast, the additional contributions $\left(t_{\lambda}\right)_{j}^{i}$ and $u^{i l} K_{l j}$ are already finite before the holographic renormalization is performed.

In principle the correction can also be found by noting that the variation of $K_{i j}$ under the assumption of the asymptotic expansion (2.18) is not independent from the variation of $\gamma_{i j}$. We note that in the case of holographic pure gravitational anomalies dual to two dimensional field theories a similar correction has been found in [24].

This definition of the stress tensor, together with the further contribution to the Ward identity (2.20) are nontrivial consequences of the dynamics of the anomalous theory. An in depth analysis of their properties will be given in a forthcoming publication.

\section{Electric and chiral magnetic conductivities}

In this section we will compute the electric DC conductivity and the chiral magnetic and chiral vortical conductivities in linear response theory. We start with the

\subsection{DC conductivity}

We introduce the small perturbations

$$
\begin{aligned}
A_{z} & =\epsilon\left(-E t+a_{z}(u)\right), \\
g_{t z} & =\frac{\epsilon}{u} h_{t}^{z}(u) \\
g_{u z} & =\frac{\epsilon}{u} h_{u}^{z}(u)
\end{aligned}
$$

$E$ is the external electric field and the perturbations all fulfill $a_{z}(0)=h_{t}^{z}(0)=h_{u}^{z}(0)=0$, i.e. they do not introduce additional sources. The equations of motion are

$$
\begin{aligned}
0 & =\left(1+2 u^{3} J \phi^{\prime}(u)^{2}\right) h_{u}^{z}+E \frac{u \phi^{\prime}(u)}{k^{2} f(u)}\left(1-\frac{k^{2}}{2} J u\right), \\
u^{2} f(u)\left(\frac{h_{t}^{\prime z}(u)}{u}\right)^{\prime} & =\frac{k^{2}}{4}\left(1+2 u^{3} J \phi^{\prime}(u)^{2}\right) h_{t}^{z}-u^{2} \phi^{\prime}(u)\left(1-\frac{k^{2}}{2} J u\right) f(u) a_{z}^{\prime}(u), \\
0 & =\left[\left(1-\frac{k^{2}}{2} J u\right)\left(f(u) a_{z}^{\prime}(u)+\phi^{\prime}(u) h_{t}^{z}(u)\right)\right]^{\prime} .
\end{aligned}
$$

We solve these equations following [25]. Note that $h_{u}^{z}(u)$ obeys an algebraic equation. Demanding regularity of the metric we find that as one approaches the horizon

$$
2 f(u) h_{u}^{z}(u)=-h_{t}^{z}(u) .
$$

The equation for $a_{z}(u)$ is a total derivative and can be integrated. On the horizon we can compute $f(u) a_{z}^{\prime}(u)$ using the equation for $h_{t}^{z}$. Noting that the current is $J_{z}=$ $2 \lim _{u \rightarrow 0} f(u) a_{z}^{\prime}(u)$ we obtain the conductivity $\sigma_{\mathrm{DC}}=J / E$

$$
\sigma_{\mathrm{DC}}=\left(1-\frac{k^{2}}{2} J\right)\left[1+\frac{\left(1-\frac{k^{2}}{2} J\right) 4 \mu^{2}}{k^{2}\left(1+2 J \mu^{2}\right)}\right] .
$$


This expression is the dimensionless conductivity where we have set the horizon to $u_{h}=1$. It is qualitatively of the same form as the one discussed in the $A d S_{4}$ model in [17], e.g. it vanishes for $k^{2} J=2$ and can become even negative in some range of the parameters indicating an instability. We also note that for $J=0$ the dimensionless conductivity obeys a similar bound as the one proven for holographic matter in $2+1$ dimensions in [15]. Our main interest is however in the anomalous transport coefficients so we will not further investigate the properties of $\sigma_{\mathrm{DC}}$.

\subsection{Chiral magnetic conductivity}

We now introduce the magnetic field as a perturbation by

$$
\begin{aligned}
& A_{y}=\epsilon B x, \\
& A_{z}=\epsilon a_{z}(u), \\
& g_{t z}=\frac{\epsilon}{u} h_{t}^{z}(u) .
\end{aligned}
$$

The equations of motion for the perturbations are

$$
\begin{aligned}
4 \alpha B \phi^{\prime}(u)= & {\left[\left(1-\frac{k^{2}}{2} J u\right)\left(f(u) a_{z}^{\prime}(u)+\phi^{\prime}(u) h_{z}^{t}(u)\right)\right]^{\prime}, } \\
u^{2} f(u)\left(\frac{h_{t}^{\prime z}(u)}{u}\right)^{\prime}= & \frac{k^{2}}{4}\left(1+2 u^{3} J \phi^{\prime}(u)^{2}\right) h_{t}^{z}-u^{2} \phi^{\prime}(u)\left(1-\frac{k^{2}}{2} J u\right) f(u) a_{z}^{\prime}(u) \\
& -2 B \lambda f(u)\left(3 u k^{2}+16 u^{2} \phi^{\prime}(u)^{2}+12 f(u)-12\right) .
\end{aligned}
$$

The strategy to integrate these equations is as follows. First we solve the equations for $a_{z}(u)$ by writing

$$
h_{z}^{t}(u)=\frac{f(u)}{\mu} a_{z}^{\prime}(u)+\frac{4 \alpha B(u-1)}{1-\frac{k^{2}}{2} J u},
$$

where we have fixed an integration constant by demanding that $h_{z}^{t}(u)$ vanishes on the horizon. The equation for $h_{z}^{t}(u)$ is now converted into a third order equation for $a_{z}(u)$. This equation can be integrated and the remaining three integration constants can be fixed by demanding regularity of the solutions on the horizon. Due to the presence of the mass terms for the graviton fluctuation, this means in particular that $h_{z}^{t}(u)$ has to vanish on the horizon. We note that for $k^{2}=0$ this is not the case and gives rise to an additional integration constant that eventually corresponds to the choice of frame in a hydrodynamic setup. Without going into the details of the solution, we note that this procedure results in the asymptotic expansions

$$
\begin{aligned}
& a_{z}(u)=4 \alpha B \mu u+O\left(u^{2}\right), \\
& h_{t}^{z}(u)=-\left(\alpha \mu^{2}+8 \lambda \pi^{2} T^{2}-\lambda \frac{k^{2}}{2}\right) B u^{2}+O\left(u^{3}\right) .
\end{aligned}
$$

The result is almost independent of the disorder parameters $k$ and $J$. However if we apply the usual holographic dictionary and compute the energy-momentum tensor from these 
solutions we would find $T_{0 z}=4 g_{t z}^{\prime}(u=0)$ [23]. As we have argued in the previous section this corresponds only to the contribution $t_{0}^{i j}$, and we need also to calculate the corrections due to $\left(t_{\lambda}\right)^{i j}$ and $u^{i l} K_{l j}$. These can be easily calculated from the background and give

$$
\begin{aligned}
\left(t_{\lambda}\right)^{i j} & =0, \\
u^{i l} K_{l}^{j} & =2 k^{2} B \lambda \delta^{i(0} \delta^{z) j} .
\end{aligned}
$$

Using therefore the new definition of energy-momentum tensor (2.25) and assembling the components $\Theta^{i 0}$ into the energy current $\vec{J}_{E}$, we find

$$
\begin{aligned}
\vec{J} & =8 \alpha \mu \vec{B}, \\
\vec{J}_{E} & =\left(4 \alpha \mu^{2}+32 \lambda \pi^{2} T^{2}\right) \vec{B} .
\end{aligned}
$$

Taking into account (2.24) these are the usual expressions for the chiral magnetic effect in (covariant) charge and currents.

\subsection{Chiral vortical conductivity}

We will represent vorticity by introducing a gravitomagnetic field $B_{g}$ in the $z$ direction. The relation between vorticity and the gravitomagnetic field follows from observing that $\Omega^{i}=\frac{1}{2} \epsilon^{i j k} \partial_{j} u_{k}$. In the rest frame $u^{\mu}=(1,0,0,0)$, the gravitomagnetic field is the mixed space-time component of the metric in the dual field theory $d s^{2}=-d t^{2}+2 d t \vec{A}_{g} d \vec{x}+d \vec{x}^{2}$. With the gravitomagnetic field defined as $\vec{B}_{g}=\vec{\nabla} \times \vec{A}_{g}$, it follows $\vec{B}_{g}=2 \vec{\Omega}$. The ansatz for the perturbations is

$$
\begin{aligned}
A_{y} & =\epsilon B_{g} u \mu x, \\
A_{z} & =\epsilon a_{z}(u), \\
g_{t y} & =\epsilon \frac{f(u)}{u} B_{g} x, \\
g_{t z} & =\epsilon \frac{1}{u} h_{t}^{z}(u) .
\end{aligned}
$$

We note that the gravitomagnetic field produces a current due to drag when the chemical potential does not vanish. The equations of motion for the perturbations are

$$
\begin{aligned}
4 \alpha B_{g} u \phi^{\prime}(u)^{2}+B_{g} \lambda & \left(20 f^{\prime}(u)^{2}-16(f(u)-1) \frac{f^{\prime}(u)}{u}+\frac{16}{3} u^{2} f^{\prime}(u) \phi^{\prime}(u)^{2}\right) \\
= & {\left[\left(1-\frac{k^{2}}{2} J u\right)\left(f(u) a_{z}^{\prime}(u)+\phi^{\prime}(u) h_{z}^{t}(u)\right)\right]^{\prime} } \\
u^{2} f(u)\left(\frac{h_{t}^{\prime z}(u)}{u}\right)^{\prime}= & \frac{k^{2}}{4}\left(1+2 u^{3} J \phi^{\prime}(u)^{2}\right) h_{t}^{z}-u^{2} \phi^{\prime}(u)\left(1-\frac{k^{2}}{2} J u\right) f(u) a_{z}^{\prime}(u) \\
& -\lambda B_{g} \mu f(u)\left(80 f(u)+17 u k^{2}+(172 / 3) u^{3} \mu^{2}-80\right) .
\end{aligned}
$$


The strategy to integrate these equations is the same as before. First we solve the equations for $a_{z}(u)$ by writing

$$
\begin{aligned}
h_{z}^{t}(u)= & \frac{f(u)}{\mu} a_{z}^{\prime}(u)+32 B_{g} \frac{1-u}{1-\frac{k^{2}}{2} J u}\left(\left(-16 u^{2}-16 u-16-144 u^{4}+48 u^{3}\right) \lambda \mu^{3}\right. \\
& +\left((-u-1) 72 \alpha+\left(576 u^{3}-24 u k^{2}-144 u^{3} k^{2}+192 u^{2}-24 k^{2}+192 u+24 u^{2} k^{2}+192\right) \lambda\right) \mu \\
& \left.+\frac{\lambda}{\mu}\left(-576 u-36 u^{2} k^{4}+144 k^{2}-576 u^{2}+288 u^{2} k^{2}-576-9 k^{4}+144 u k^{2}\right)\right)
\end{aligned}
$$

Again we have fixed an integration constant by demanding that $h_{z}^{t}(u)$ vanishes on the horizon. The equation for $h_{z}^{t}(u)$ is now converted into a third order equation for $a_{z}(u)$. This equation can be solved with the same boundary conditions as in the case of the magnetic field. The details of the lengthy solutions are not interesting to us. The resulting asymptotic expansions are

$$
\begin{aligned}
& a_{z}(u)=\left(2 \alpha \mu^{2} u+16 \pi^{2} T^{2} \lambda\right) B_{g} u+O\left(u^{2}\right), \\
& h_{t}^{z}(u)=-\left(\frac{2}{3} \alpha \mu^{3}+16 \lambda \mu \pi^{2} T^{2}\right) B_{g} u^{2}+O\left(u^{3}\right) .
\end{aligned}
$$

In contrast to the case with magnetic field, the asymptotic expansions are completely independent of the disorder parameters. This is accord with the form of the energymomentum tensor since the new contributions to it depend only on external electromagnetic fields but not on the externally applied gravitomagnetic field. We find thus for the response due to a gravitomagnetic field

$$
\begin{aligned}
\vec{J} & =\left(4 \alpha \mu^{2}+32 \pi^{2} T^{2} \lambda\right) \vec{B}_{g}, \\
\vec{J}_{E} & =\left(\frac{8}{3} \alpha \mu^{3}+64 \lambda \mu \pi^{2} T^{2}\right) \vec{B}_{g} .
\end{aligned}
$$

Remembering that gravitomagnetic field and vorticity are related by $\vec{B}_{g}=2 \vec{\Omega}$, these are the usual responses of a chiral fluid due to vorticity.

\section{Conclusion and discussion}

Chiral magnetic and chiral vortical effects are anomaly induced dissipationless transport phenomena. Holography has played a major role in their discovery and interpretation. Here we showed that - as expected - in a simple holography model of disorder the chiral magnetic and chiral vortical effects are unchanged.

While this result could have been expected and at least the response in the charge current could be inferred from [26], the response in the energy current turned out to be more interesting. The mixed gauge gravitational Chern-Simons term modifies the definition of the holographic energy-momentum tensor. We found the new form by allowing the extrinsic curvature to vary independently near the boundary. Then we demanding that 
the energy momentum tensor fulfills a standard Ward identity in which the extrinsic curvature acts like an external source conjugate to the operator $u^{i j}$. We emphasize however that the background we found is a perfectly asymptotically AdS background in which the extrinsic curvature is asymptotically proportional to the induced metric in the standard fashion $K_{i j}=\gamma_{i j}+\ldots$. Nevertheless, even to first order in the gravitational Chern-Simons coupling $\lambda$, the new terms in the energy-momentum tensor contribute and precisely restore the usual form of the chiral magnetic effect in the energy current. It is also important to realize that the new terms contribute only due to the fact that the asymptotic expansion of the metric contains a constant term, $g_{t t}=-\frac{1}{u}+\frac{k^{2}}{4}+O(u)$ and $g_{u u}=\frac{1}{4 u}+\frac{k^{2}}{16}+O(u)$, which is a direct consequence of the presence of a background of massless scalar fields.

Another important remark concerns the form of the anomalous transport coefficients. In translation symmetry preserving backgrounds, an additional integration constant in the solution for the fluctuation $h_{t}^{z}$ is allowed. This integration constant can then be used to choose a frame, e.g. one could use it to fix the Landau frame [27]. In the theory with a massive graviton, such an integration constant is absent and regularity of the solution uniquely fixes the frame which should be interpreted as the disorder rest frame [20].

We also note that the gravitational Chern-Simons term and the charge disorder term proportional to the coupling $J$ are both of higher derivative nature and therefore should in principle be understood as perturbative couplings in an effective field theory manner, viewing them as subleading terms in the $1 / N$ expansion. Since we are only interested in linear response in the magnetic and gravito-magnetic fields, this is automatic for the Chern-Simons coupling $\lambda$. We decided however to follow $[16,17]$ who treat the analogues of the $J$ coupling non-perturbatively. This is interesting since it allows to compare the DC conductivity (3.8) with the ones obtained there. We note that there is an unphysical region in the parameter space in which the DC conductivity becomes negative. It is remarkable that the anomalous transport coefficients can be computed exactly for all values of $J$ and are indeed independent of it.

It is also interesting to recall the results of [28] in which a Stückelberg field was added to the gauge sector, thereby breaking gauge invariance in the bulk. In this theory the chiral magnetic conductivity did get corrected. In contrast the Stückelberg field giving a mass to the graviton does not influence the anomalous conductivities. Intuitively we propose to understand that as follows. The massive gravity theory breaks spatial translation invariance. There is however no anomalous conductivity related to the conserved momentum. The corresponding currents would be the purely spatial components of the energy momentum tensor. Threfore the anomalous terms in $\Theta^{0 i}$ should be interpreted as energy current and not as the (non-conserved) momentum density. In our setup energy and charge are still conserved up to the anomalies. The anomalous transport in energy and charge current is not modified. In this line of reasoning it would be very interesting to study holographic setups that implement energy non-conservation.

Finally we note that the new form of the energy-momentum tensor should be useful to understand the effects of the mixed gravitational Chern-Simons term on transport as has been recently studied for other higher derivative actions in [29]. 


\section{Acknowledgments}

We would like to thank O. Pujolàs, K. Schalm, Y. Liu, M. Baggioli and M. Valle for useful discussions. This research has been supported by Plan Nacional de Altas Energías Spanish MINECO grants FPA2015-65480-P and FPA2015-64041-C2-1-P, by the Basque Government grant IT979-16, and by the Centro de Excelencia Severo Ochoa Programme under grant SEV-2012-0249. The work of J.F.-P. is supported by fellowship SEV-20120249-03. The work of C.C. is funded by Fundación La Caixa under "La Caixa-Severo Ochoa" international predoctoral grant. The work of E.M. is supported by the Universidad del País Vasco UPV/EHU, Bilbao, Spain, as a Visiting Professor.

Open Access. This article is distributed under the terms of the Creative Commons Attribution License (CC-BY 4.0), which permits any use, distribution and reproduction in any medium, provided the original author(s) and source are credited.

\section{References}

[1] D.E. Kharzeev, The Chiral Magnetic Effect and Anomaly-Induced Transport, Prog. Part. Nucl. Phys. 75 (2014) 133 [arXiv:1312.3348] [inSPIRE].

[2] K. Landsteiner, Notes on Anomaly Induced Transport, in proceedings of the 56th Cracow School of Theoretical Physics: A Panorama of Holography, Zakopane, Poland, 24 May-1 June 2016 [Acta Phys. Polon. B 47 (2016) 2617] [arXiv: 1610.04413] [INSPIRE].

[3] G.M. Newman, Anomalous hydrodynamics, JHEP 01 (2006) 158 [hep-ph/0511236] [INSPIRE].

[4] N. Banerjee, J. Bhattacharya, S. Bhattacharyya, S. Dutta, R. Loganayagam and P. Surowka, Hydrodynamics from charged black branes, JHEP 01 (2011) 094 [arXiv:0809. 2596] [INSPIRE].

[5] J. Erdmenger, M. Haack, M. Kaminski and A. Yarom, Fluid dynamics of R-charged black holes, JHEP 01 (2009) 055 [arXiv:0809.2488] [INSPIRE].

[6] K. Landsteiner, E. Megias, L. Melgar and F. Pena-Benitez, Holographic Gravitational Anomaly and Chiral Vortical Effect, JHEP 09 (2011) 121 [arXiv:1107.0368] [INSPIRE].

[7] M. Ammon and J. Erdmenger, Gauge/gravity duality, Cambridge University Press, Cambridge U.K. (2015).

[8] J. Zaanen, Y.-W. Sun, Y. Liu and K. Schalm, Holographic Duality in Condensed Matter Physics, Cambridge University Press, Cambridge U.K. (2015).

[9] D. Vegh, Holography without translational symmetry, arXiv:1301.0537 [INSPIRE].

[10] R.A. Davison, Momentum relaxation in holographic massive gravity, Phys. Rev. D 88 (2013) 086003 [arXiv: 1306.5792] [INSPIRE].

[11] M. Blake and D. Tong, Universal Resistivity from Holographic Massive Gravity, Phys. Rev. D 88 (2013) 106004 [arXiv: 1308.4970] [inSPIRE].

[12] M. Blake, D. Tong and D. Vegh, Holographic Lattices Give the Graviton an Effective Mass, Phys. Rev. Lett. 112 (2014) 071602 [arXiv:1310.3832] [INSPIRE]. 
[13] A. Donos and J.P. Gauntlett, Holographic Q-lattices, JHEP 04 (2014) 040 [arXiv: 1311.3292] [INSPIRE].

[14] T. Andrade and B. Withers, A simple holographic model of momentum relaxation, JHEP 05 (2014) 101 [arXiv:1311.5157] [INSPIRE].

[15] S. Grozdanov, A. Lucas, S. Sachdev and K. Schalm, Absence of disorder-driven metal-insulator transitions in simple holographic models, Phys. Rev. Lett. 115 (2015) 221601 [arXiv: 1507.00003] [INSPIRE].

[16] M. Baggioli and O. Pujolàs, On holographic disorder-driven metal-insulator transitions, JHEP 01 (2017) 040 [arXiv:1601.07897] [INSPIRE].

[17] B. Goutéraux, E. Kiritsis and W.-J. Li, Effective holographic theories of momentum relaxation and violation of conductivity bound, JHEP 04 (2016) 122 [arXiv:1602.01067] [INSPIRE].

[18] E. Megias, Anomalous transport and massive gravity theories, J. Phys. Conf. Ser. 804 (2017) 012029 [arXiv:1701.00085] [inSPIRE].

[19] I. Amado, K. Landsteiner and F. Pena-Benitez, Anomalous transport coefficients from Kubo formulas in Holography, JHEP 05 (2011) 081 [arXiv: 1102.4577] [INSPIRE].

[20] M.A. Stephanov and H.-U. Yee, No-Drag Frame for Anomalous Chiral Fluid, Phys. Rev. Lett. 116 (2016) 122302 [arXiv:1508.02396] [InSPIRE].

[21] W.A. Bardeen and B. Zumino, Consistent and Covariant Anomalies in Gauge and Gravitational Theories, Nucl. Phys. B 244 (1984) 421 [InSPIRE].

[22] E. Megias and F. Pena-Benitez, Holographic Gravitational Anomaly in First and Second Order Hydrodynamics, JHEP 05 (2013) 115 [arXiv:1304.5529] [INSPIRE].

[23] S. de Haro, S.N. Solodukhin and K. Skenderis, Holographic reconstruction of space-time and renormalization in the AdS/CFT correspondence, Commun. Math. Phys. 217 (2001) 595 [hep-th/0002230] [INSPIRE].

[24] P. Kraus and F. Larsen, Holographic gravitational anomalies, JHEP 01 (2006) 022 [hep-th/0508218] [INSPIRE].

[25] A. Donos and J.P. Gauntlett, Novel metals and insulators from holography, JHEP 06 (2014) 007 [arXiv: 1401.5077] [INSPIRE].

[26] S. Grozdanov and N. Poovuttikul, Universality of anomalous conductivities in theories with higher-derivative holographic duals, JHEP 09 (2016) 046 [arXiv: 1603.08770] [INSPIRE].

[27] D.T. Son and P. Surowka, Hydrodynamics with Triangle Anomalies, Phys. Rev. Lett. 103 (2009) 191601 [arXiv:0906.5044] [INSPIRE].

[28] A. Jimenez-Alba, K. Landsteiner and L. Melgar, Anomalous magnetoresponse and the Stückelberg axion in holography, Phys. Rev. D 90 (2014) 126004 [arXiv:1407.8162] [INSPIRE].

[29] A. Donos, J.P. Gauntlett, T. Griffin and L. Melgar, DC Conductivity and Higher Derivative Gravity, Class. Quant. Grav. 34 (2017) 135015 [arXiv:1701.01389] [INSPIRE]. 